PCB and DDE and child motor development have found contradicting results. The aim of this follow-up study was to examine the association between prenatal exposure to DDE and PCB and motor development and developmental milestones; crawling, standing-up and walking in children in Greenland, Ukraine and Poland.

Methods CB-153 and p,p'-DDE were measured in maternal blood in second or third trimester of pregnancy as a bio-marker of the child's prenatal exposure to the compounds. A total of 1,103 children aged 5 to 9 years were followed up in 20102012. Motor development were measured in terms of the parentally assessed screening tool Developmental Coordination Disorder Questionnaire 2007 (DCDQ'07) and developmental milestones were assessed via parental reports of child age at the first time of crawling, standing up and walking. The association between $\mathrm{PCB} / \mathrm{DDE}$ and motor skills and milestones were analysed by means of linear multiple regression analyses using tertiles of exposure and stratified by country. Both complete case analyses and multiple imputation based analyses were executed. Adjustment were performed for the co-variates; maternal age, maternal smoking during pregnancy, maternal alcohol before pregnancy, maternal education, parity, gestational age at blood sampling, preterm birth, breastfeeding, child sex and child age at interview.

Results We found no associations between prenatal PCB and DDE exposure and developmental milestones or motor skills. Complete case- and multiple imputation based analyses showed adjusted mean differences in motor skills and age at milestones around null, in all three countries.

Conclusions These results on 1.103 mother-children-pairs from the INUENDO cohort in Greenland, Warsaw (Poland) and Kharkiv (Ukraine), indicate no association between in utero PCB/ DDE exposure and developmental milestones and motor skills.

\section{EXPOSURE TO POLYCYCLIC AROMATIC HYDROCARBONS AND SPERM QUALITY OF COKE-OVEN WORKERS}

${ }^{1} \mathrm{~W}$ Y Lin, ${ }^{2}$ Jeng, ${ }^{3} \mathrm{Pan},{ }^{4} \mathrm{M}$ L Yu, ${ }^{4} \mathrm{C}$ Y Dai, ${ }^{1} \mathrm{C} \mathrm{H} \mathrm{Li},{ }^{1} \mathrm{~N}$ C Chang, ${ }^{5}$ Huang, ${ }^{4} \mathrm{M}$ H Hsieh, ${ }^{4} \mathrm{~J} \mathrm{~F}$ Yang, ${ }^{4} \mathrm{C}$ L Wang, ${ }^{6} \mathrm{C}$ F Huang, ${ }^{7}$ Lin, ${ }^{4} \mathrm{H}$ Y Chuang, ${ }^{1} \mathrm{M}$ T Wu, ${ }^{8} \mathrm{C}$ K Ho. ${ }^{1}$ Kaohsiung Municipal Hsiaokang Hospital, Kaohsiung, Taiwan; ${ }^{2}$ School of Community and Environmental Health, College of Health Sciences, Old Do, Norfolk, United States of America; ${ }^{3}$ nstitute of Occupational Safety and Health, Council of Labor Affairs, Taipei, Taiwan; ${ }^{4}$ Kaohsiung Medical University Hospital, Kaohsiung, Taiwan; ${ }^{5}$ Department of Occupational Medicine, Kaohsiung Municipal Hsiaokang Hospital, KMU, Kaohsiung, Taiwan; ${ }^{6}$ Department of Occupational Medicine, Kaohsiung Municipal Ta-Tung Hospital, Kaohsiung, Taiwan; ${ }^{7}$ China Steel Corporation, Kaohsiung, Taiwan; ${ }^{8}$ Department of Health, Kaohsiung City Government, Kaohsiung, Taiwan

\subsection{6/oemed-2013-101717.224}

Objectives The study aimed to assess whether exposure to polycyclic aromatic hydrocarbons (PAHs) alter sperm quality of coke-oven workers.

Methods Personal breathing zone, urine, and semen samples were collected from nonsmoking workers at top-side ovens (high exposure, $\mathrm{N}=16$ ) and side-ovens (low exposure, $\mathrm{N}=20$ ), and administrative staff members serving as the control group $(\mathrm{N}=$ 15). PAH concentrations were analysed by a gas chromatography quadruple spectrometer. Routine semen was analysed by procedures in accordance with the World Health Organization guidelines. Urinary 1-hydroxypyrene (1-OHP) was analysed by HPLC with a fluorescence detector.

Results ANOVA analysis showed a significant difference in urinary 1 -OHP levels $(14.7 \pm 12.9,4 \pm 4.3,0.3 \pm 0.2 \mathrm{~g} / \mathrm{g}$ creatinine respectively, $\mathrm{p}=0.02$ ) between the exposed groups and the control. Mean concentrations of 16 species of PAHs significantly differed among the three groups (Total PAHs $=41620.3 \pm$ $17697.6 \mathrm{ng} / \mathrm{m}^{3}, 19887.6 \pm 1378.1 \mathrm{ng} / \mathrm{m}^{3}, 57.0 \pm 18.1 \mathrm{ng} / \mathrm{m}^{3}$, $\mathrm{p}<0.0001$; Benzo (g,h,i)perylene $=3553.9 \pm 1250.7 \mathrm{ng} / \mathrm{m}^{3}$, $3001.9 \pm 1367.7 \mathrm{ng} / \mathrm{m}^{3}, 2.0 \pm 1.5 \mathrm{ng} / \mathrm{m}^{3}, \mathrm{p}<0.0001, \mathrm{p}=$ 0.067). Asthenospermia was found more frequently in the high exposure group as compared to the control $(37.5 \%$ and $13.3 \%$, respectively, $\mathrm{p}<0.01$ ). The exposed groups had lower percentages of normal morphology as compared with the control group $(14.5 \pm 3.4 \%, 15.0 \pm 3.1 \%$, $34.5 \pm 2.6 \%$ $\mathrm{p}<0.01)$. Multiple regression analysis showed that PAH species positively correlated with abnormality of morphology and motility of sperm.

Conclusion Abnormal morphology of sperm was associated with $\mathrm{PAH}$ concentrations, especially those with heavy molecular weights, e.g. benzo (g,h,i)perylene and benzo (k)fluoranthene.

\section{EXPOSURE TO POLYBROMINATED DIPHENYL ETHERS AND MALE REPRODUCTIVE FUNCTION IN ARCTIC AND EUROPEAN POPULATIONS}

${ }^{1} \mathrm{G}$ T Toft, ${ }^{2}$ Lenters, ${ }^{2}$ Vermeulen, ${ }^{2}$ Heederik, ${ }^{3}$ Thomsen, ${ }^{3}$ Becher, ${ }^{4}$ Giwercman, ${ }^{5}$ Bizzaro, ${ }^{6}$ Manicardi, ${ }^{7}$ Spanó, ${ }^{8}$ Rylander, ${ }^{9}$ Pedersen, ${ }^{10}$ Strucinski, ${ }^{11}$ Zviezdai, ${ }^{12}$ Bonde. ${ }^{1}$ Aarhus University Hospital, Aarhus, Denmark; ${ }^{2}$ Institute for Risk Assessment Sciences, Utrecht University, Utrecht, Nederland; ${ }^{3}$ Department of Chemistry, University of Oslo, Oslo, Norway; ${ }^{4}$ Reproductive Medicine Centre, Skåne University Hospital, Malmö, Sweden; ${ }^{5}$ Department of Life and Environmental Sciences, Polytechnic University of Marche, Ancona, Italy; ${ }^{6}$ Department of Life Science, Università di Modena e Reggio Emilia, Reggio Emilia, Italy; 'Unit of Radiation Biology and Human Health, ENEA Casacia, Rome, Italy: ${ }^{8}$ Division of Occupational and Environmental Medicine, Lund University, Lund, Sweden; ${ }^{9}$ Centre for Arctic Environmental Medicine, Nuuk, Greenland; ${ }^{10}$ Department of Toxicology and Risk Assessment, Warsaw, Poland; ${ }^{11}$ Kharkiv National Medical University, Kharkiv, Ukraine; ${ }^{12}$ Bispebjerg University Hospital, Copenhagen, Denmark

\subsection{6/oemed-2013-101717.225}

Background Animal and a few human studies suggest that polybrominated diphenyl ethers (PBDEs) may affect male reproductive function. The aim of the present study was to evaluate if male reproductive function was associated with serum levels of PBDEs.

Methods We evaluated the effects of environmental exposure to BDE-28, BDE-47 and BDE-153 on reproductive hormones and semen quality, including markers of DNA damage and apoptosis, in 299 men from Greenland, Poland and Ukraine.

Results Adjusted linear regression models indicated that sperm DNA damage measured by the TUNEL assay increased by $0.22 \%$, confidence interval (CI) $0.03 \%$ to $0.42 \%$ for each percentage increase in lipid adjusted BDE-47 concentration, and semen volume decreased by $0.11 \%(0.01 \%$ to $0.19 \%)$ for each percentage increase in BDE-28 exposure.

Conclusions Adverse effects of PBDE exposure on semen volume and sperm DNA damage were observed but other conventional semen parameters and reproductive hormones were not affected. Harmful effects of PBDE exposure on sperm DNA damage is supported by experimental evidence based on other cell types.

\section{PRENATAL BLOOD LEAD LEVEL AND CHILDHOOD NEUROBEHAVIORAL DEFICIT}

${ }^{1} \mathrm{M}$ V Vigeh, ${ }^{2}$ Yokoyama, ${ }^{2}$ Matsukawa, ${ }^{3}$ Shinohara, ${ }^{4}$ Shahbazi, ${ }^{1}$ Ohtani. ${ }^{1}$ National Institute of Occupational Safety and Health, Kawasaki, Japan; ${ }^{2} J$ untendo University Faculty of Medicine, Tokyo, Japan; ${ }^{3}$ Seisen University, Tokyo, Japan; ${ }^{4}$ Tehran University of Medical Sciences, Tehran, Iran

10.1136/oemed-2013-101717.226 
Objectives Lead is one of the oldest known toxic metals. For decades, its effects on child development has been remained a topic of concern with an increased interest in 'what prenatal blood lead levels should be considered toxic'. Many resent studies have shown the impacts of increased blood lead on different aspects of infants' development at 'acceptable' levels $(\leq 100 \mu \mathrm{g} / \mathrm{L})$.

Methods To investigate the effects of prenatal lead exposure on children mental development, we have conducted a longitudinal study. Pregnant women $(n=364)$ who referred to hospitals for prenatal care at the first trimester of pregnancy were asked to participate in the survey. Maternal whole blood (MWB) samples, one for each pregnancy trimesters (3 times), and the umbilical cord blood samples, at the time of delivery, were collected and subjected to ICP-MS analysis for measurement of lead concentrations. We invited the mothers and their children to the research hospitals when the children were between 20 and 36 months of age and assessed mental development using Early Child Development Inventory (ECDI). The inventory included 60 items, which cover seven different development areas.

Results MWB lead followed a U-shaped pattern over the course of pregnancy with lowest level during the second trimester. The ECDI score was inversely related to the first trimester blood lead concentrations $(r=-0.15, \mathrm{p}<0.05)$. The logistic regression analysis demonstrated significant relationships between increasing the first trimester lead concentrations $\left(\log _{\mathrm{e}}\right)$ with low score of ECDI, adjusting for multiple covariates (Unit risk: 5.7, 95\% CI: $1.1-30.7, \mathrm{p}<0.001)$.

Conclusions Increased prenatal lead concentrations, even at "acceptable" level, adversely affects ECDI scores. Therefore, a reappraisal of lead exposure standards for female workers is a critical public health concern.

\section{CORD BLOOD MERCURY, APOE AND CHILD'S BEHAVIOUR}

${ }^{1} \mathrm{~S} \mathrm{Ng},{ }^{1}$ Lin, ${ }^{2}$ Jeng, ${ }^{1} \mathrm{H}$ wang, ${ }^{2} \mathrm{H}$ sieh, ${ }^{1} \mathrm{C}$ Chen. ${ }^{1}$ Institute of Occupational Medicine and Industrial Hygiene, Taipei, Taiwan; ${ }^{2}$ National Taiwan University College of Medicine, Taipei, Taiwan

\subsection{6/oemed-2013-101717.227}

Objective Mercury $(\mathrm{Hg})$ is recognised as neurotoxin; nevertheless, the effect of prenatal mercury exposure on child behavior in fish eating population is still controversial. The benefit of nutrient element of fish may insufficient to explain it. Apolipoprotein (APOE) is a major protein transporter in brain, epsilon 4 (e4) allele is recognised with poorer neural repair function. We hypothesize that the APOE may modifier the effect of prenatal mercury exposure on child behaviour.

Methods The present study is a prospective cohort study. There were 166 subjects recruited at delivery and followed up at age of two years. The level of prenatal mercury exposure is determined in cord blood and the genotype of APOE is analysis by the methods Restriction Fragment Length Polymorphism Analysis of PCR-Amplified Fragments (PCR-RFLP). The Child Behavior Checklists version $1.5 / 5$, a parent rating scale, is used to determine the child's behaviour.

Results The adverse effect is found in e4 carriers whose cord blood $\mathrm{Hg}$ level is greater than $12 \mathrm{ug} / \mathrm{l}$. After controlling for the potential confounding factors, the total scale of internalising behavior $(\mathrm{b}=8.4)$ and all symptoms of internalising problems is found statistically significant higher in this group. The symptoms and beta coefficients are emotional problem $(b=2.6)$, anxiety/ depression $(b=2.4)$, somatic complaints $(b=1.68)$ and withdrawn $(b=1.7)$. In additional to internalising behaviour, the item of other problem $(b=6.7)$ from externalising behaviour and the total scale of CBCL $(b=20.7)$ are also found statistically significant higher in the group that e4 carriers with greater cord blood $\mathrm{Hg}$.

Conclusion APOE gene modifies the effect of prenatal mercury exposure on neurobehaviour. The different frequency of gene susceptible across populations may be a reason of the controversial finding in previous study. The impact of genetic susceptibility should be considered in future study.

\section{Session: 1. Health impact analysis I}

\section{OTOTOXICITY DUE TO LOW CONCENTRATION EXPOSURE TO SOLVENT MIXTURES IN PAINT FACTORY WORKERS}

${ }^{1}$ C A J P Juarez Perez, ${ }^{1}$ A T V Torres-Valenzuela, ${ }^{2}$ L C H G Haro-Garcia, ${ }^{1}$ V H Borja-Aburto, ${ }^{1} \mathrm{G}$ A M Aguilar-Madrid. 'Mexican Social Security Institute, Distrito Federal, Mexico; ${ }^{2}$ University of Mexico City, Distrito Federal, Mexico

\subsection{6/oemed-2013-101717.228}

Objective To evaluate auditory dysfunction in workers exposed to low doses of solvent mixtures.

Methods 77 workers exposed to solvents as well as a control group of 84 non-exposed individuals were studied. Fourteen solvents were measured, including toluene, xylene, and n-hexane. Pure tone audiometries were performed at $125 \mathrm{~Hz}$ to $8 \mathrm{KHz}$, as well as brainstem evoked response.

Results In the exposed group, subjects' hearing decreased in the measured frequencies, in both ears. Robust multiple linear regression models for frequency means between $125 \mathrm{~Hz}$ and 8 $\mathrm{KHz}$, for the left ear $R^{2}=33.3 \%$, exposed $\beta=4.1(p<0.001)$ and for the right ear $R^{2}=38 \%$, exposed $\beta=4.8 \quad(p<0.001)$, adjusted by age, chronic pathologies and environmental noise exposure. There was major latency in waves III and V and in intervals I-V and I-III $(p<0.05)$, in both ears, adjusted for age and chronic pathologies.

Conclusions It is likely that low exposures to solvent mixtures affect hearing and their central pathways. It is important to elucidate whether this affection could indicate incipient ototoxicity and neurotoxicity.

\section{HOW WELL ARE WE CONTROLLING FALLS FROM HEIGHT IN CONSTRUCTION EXPERIENCES OF UNION} CARPENTERS IN WASHINGTON STATE, USA, 1989-2008

H J Lipscomb. Duke University Medical Center, Durham NC, United States of America

\subsection{6/oemed-2013-101717.229}

Objective By linking data on union work hours with workers' compensation records, rates of reported work-related injuries resulting from $\mathrm{FFH}$ and associated days away from work were evaluated among a large cohort $(n=24,830)$ of union carpenters in Washington State from 1989 to 2008.

Methods Using Poisson regression we assessed rates of FFH over the 20-year period while adjusting for temporal trend in work-related injuries that did not involve a FFH. Patterns of paid lost days were assessed with negative binomial regression.

Results Crude rates of FFH decreased $82 \%$ over the 20 -year period in a fairly steady pattern. Reductions were more modest and without demonstrable change since 1996 when considering the temporal reduction in non-FFH injuries. Younger workers 